\title{
Walking on water
}

\section{STEPHEN CHILDRESS}

Applied Mathematics Laboratory, Courant Institute of Mathematical Sciences, New York University, New York, NY 10012, USA

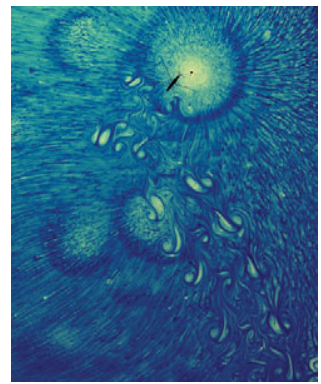

Many insects and spiders can travel on the water surface by rapid synchronized movements of the legs. It has been found that frictional forces, capillary waves and the creation of water jets and eddies beneath the fluid surface are all involved in the mechanisms of propulsion used by these creatures. Elaborate adaptations of the body structure mediate the wetting and de-wetting of the body surface to provide support and differential adhesion. Flow visualization as well as theoretical modelling have led to an understanding of the mechanisms invoked by water striders and spiders for water walking with a variety of gaits and speeds.

\section{Introduction}

In the late fifteenth century Leonardo da Vinci conceived of a device intended to enable a human to walk on water. The surviving sketch shows a man wearing a set of pontoon-like shoes, and carrying a pair of similarly equipped walking sticks. The rear leg is poised as if digging into the water, the stance resembling that of a cross-country skier. Although the shoes appear to be far too small to provide the necessary buoyancy, da Vinci has clearly dealt with the two main problems of this kind of locomotion, namely a means of providing the necessary support and stability, and, less obviously, a mechanism for generating sufficient horizontal force to propel the body forwards. Although his idea has been elaborated in over 100 patents in the last 150 years, human water walking does not seem to have attracted many adherents.

On the smaller scale of the insect world, however, walking on water is a widespread and highly developed activity. Surface tension becomes dominant for both support and locomotion, and the nature of the contact between insect and fluid interface assumes importance for both static and dynamic balance. The fluid dynamical basis for this movement is the subject of the work of Hu \& Bush (J. Fluid Mech., 2010, this issue, vol. 644, pp. 5-33), who build a theoretical framework for explaining various techniques of water walking, on the basis of extensive experiments with water-walking and terrestrial insects and their previous studies (Bush \& $\mathrm{Hu} 2006$ ).

Water striders and various spiders can be seen scurrying about on most ponds, achieving speeds in excess of a metre per second, so fast that the details of the motion are not resolvable with the naked eye. It is only in the last thirty years that high-speed photography has enabled a close look at the mechanisms at work. Capillary waves, momentum carried by vortex structures in the fluid, and viscous and contact forces at the interface may contribute to propulsion. An analogy closer to our human scale helps to understand the challenge faced by the water strider. Imagine a swimming pool 
with a slippery elastic sheet stretched tight at the water surface, enabling a human to crouch on hands and feet without dimpling too much the surface. In the absence of any friction, one could attempt to 'get a grip' on the water, driving the dimpled membrane beneath the feet backwards, either to generate fluid-elastic waves or else to create eddies in the subsurface water which could carry the momentum necessary for forward motion. This analogy omits the problem of wetting and de-wetting of the legs, important issues for the insect. But there are clearly a number of possibilities for locomotion. The question is, what do the insects do?

\section{Overview}

The water-walking insects and spiders belong to the phylum of Arthropods, animals with articulated bodies and limbs. The water walkers support themselves on the surface by being water-repellent, and the principles of the static equilibrium are well understood (Keller 1998). They propel themselves almost always by the movement of their legs. (Some semi-aquatic insects can secrete a surfactant to create a propulsive surface tension gradient. Others can climb menisci by creating a suitable static surface deformation.) It has been recognized for some time that a variety of gaits are used, including the movement of the legs as oars in a sculling motion (Suter et al. 1997). Hu \& Bush (2010) carry out experimental studies on six water-walking and twenty terrestrial insects and spiders. Through flow visualization and high-speed photography, flow patterns generated by locomotion at various speeds are observed. The experiments reveal that subsurface jets and what appear on the surface as vortex dipoles are usually generated by water walkers, often along with capillary wave packets. The partition of momentum between vortices and waves has been much debated, but these experiments show that subsurface vortex dipoles usually dominate over waves. Another mode of locomotion, seen for example in young water striders, involves a 'brushing' of the water, with little deformation of the surface.

But the microstucture of the 'integument' (i.e. the surface layer or 'skin' of the organism) is also used for the transmission of force to the free surface (Bush, Hu \& Prakash 2008). The forces which matter are the normal pressure force, contact forces associated with the partial wetting of the integument, and viscous forces associated with the brushing of the legs along the surface. The tilt and shape of the hairs of the more successful water walkers emphasize contact over viscous forces. Nevertheless viscous forces realized by the brushing of the legs is available to all of the walkers, and in particular to small arthropods such as baby water striders. The contact force results from the difference between the contact angles at the advancing and retreating parts of the integument, or 'contact angle hysteresis'. Thus, on microscales the leg can be viewed as tugging on the underlying fluid, rather than digging into it. Estimates reveal that the observed hairs are capable of generating contact and viscous forces sufficiently large to account for the observed locomotion.

Macroscopically, the legs of these arthropods are the oars which drive them forwards. They deform the surface, giving rise to curvature forces created by the surface tension. Asymmetric deformation of the surface gives rise to horizontal forces and thus to backward momentum in the subsurface water. By conservation of momentum the insect body moves forwards. The water striders provide the most dramatic example of the rowing gait. They have three pairs of legs, the middle pair being the driving legs. The driving stroke of the approximately $1 \mathrm{~cm} \mathrm{leg} \mathrm{lasts} \mathrm{about}$ $1 / 100$ of a second. As the leg moves downwards and backwards, the depth of the meniscus of the leg tip is about $1 / 10 \mathrm{~cm}$. Estimates indicate that the momentum 
carried by the resulting capillary wave packets is an order of magnitude less than that carried by the vortical structures.

The fisher spider uses a similar rowing gait with the leading three pairs of legs (Suter et al. 1997). To move quickly, however, fisher spiders transition to a galloping gait, with a deeper meniscus and a plunging downward movement of the legs. Water striders and spiders can also leap off the water to the height of several body lengths, and often the recoil from the driving stroke in the rowing gait lifts the body into the air.

For the fluid dynamicist, the most interesting theoretical issues raised by these observations are concerned with the mechanism of thrust generation by the driving legs in the rowing gait, and the resulting reaction of the subsurface water. In the world of animal locomotion, water walkers share the property of birds, fish and most flying insects of having a fairly large Reynolds number, approximately 70 based on the leg width and 100 times greater based upon leg length. In this regime of locomotion, the mechanisms of propulsion are typically based upon inertial forces and involve shed vorticity and vortical wakes (Lighthill 1975).

At the macroscopic level, most water walkers are moving in a parameter regime of small Weber number, $W e=\rho U^{2} w / \sigma$, and also small Bond number, $B o=\rho g h w / \sigma$, where $U, w, h$ represent the walking speed, the width of the leg pressing against the meniscus, and the average length the leg depresses the meniscus, respectively, while $\sigma$ is the surface tension $\rho$ is the density of water, and $g$ is gravity of course. Thus inertial effects and buoyancy forces are small compared to the curvature forces created by deformation of the fluid surface. The body is driven forwards by the appropriate component of the surface tension force, integrated over the deformed surface. The subsurface fluid acquires equal and opposite momentum, a fact that is verified experimentally here by $\mathrm{Hu} \&$ Bush (2010), by using estimates appropriate to dipoles and wave packets. Note that the deformation of the surface requires that the leg velocity exceed the capillary wave speed of $23 \mathrm{~cm} \mathrm{~s}^{-1}$. Walkers unable to meet this condition are found to use the 'brushing' technique, relying on the microscopic contact and viscous forces without significant surface deformation.

How is the backward momentum carried by the fluid? One useful idealization is to consider a concentrated, impulsive force distributed over a region just below the fluid surface. This problem was considered by Bühler (2007) in the context of water strider locomotion. If $\boldsymbol{F}$ denotes the localized force field, the identities

$$
\int \boldsymbol{F} \mathrm{d} V=-\int \boldsymbol{x} \nabla \cdot \boldsymbol{F} \mathrm{d} V=\frac{1}{2} \int \boldsymbol{x} \times(\nabla \times \boldsymbol{F}) \mathrm{d} V,
$$

obtained in three dimensions by integration by parts, show that $\boldsymbol{F}$ must be the sum of potential and solenoidal vortical parts, whose first moments are in the ratio 1:2. These moments, Bühler demonstrates, determine the momentum carried to the far field by the vortical part to be $2 / 3$ of the whole, while the pressure part, presumably realized in the far field by capillary wave packets, carries $1 / 3$. The observations reveal a variety of patterns including vortex dipoles and sinuous jets. One sees in particular the basic outcome of the paired leg strokes to be paired subsurface jets, which can either roll up into two separate dipoles, or else combine to produce a pair of oppositely signed eddies supporting a backward jet between them.

\section{Future}

The three-dimensional geometry of the vortical structures generated by the driving legs remains unclear. One scenario would have vortex tubes detach from the boundary 
layer, then diffuse into U-shaped vortex tubes, whose ends are observed at the surface as vortex dipoles. It would be of interest to to have this process clarified.

A related problem is the optimization of momentum production as a function of the combination of downward and backward movements of the leg. Prakash \& Bush (submitted) show that the elasticity of the integument is important to the anisotropy of the contact forces - perpendicular motion of the leg produces a far larger force than parallel motion. The mechanism for easily detaching and reattaching to the water surface, observed during leaping and the flight phase of some of the water walkers, and the role of flexibility on these behaviours, is likely to be another area of future interest. Our understanding of the mechanism used by the water-walking arthropods should guide the design of biomimetic water-walking devices, as well as novel mechanisms for fluid transport on the microscale.

Saffman (1967) analysed the locomotion of a deformable body in a perfect fluid, and showed how momentum conservation allowed such locomotion, provided that the centre of mass of the body could be adjusted relative to the centre of volume. Here, locomotion depends upon the body shape affecting the body's virtual mass. To the extent that frictional forces may be disregarded, water walking by arthropods is locomotion on a perfect fluid. It should be possible to analyse the process in a similar way, at least in part, as 'recoil' locomotion. For the water strider, deformation of the water surface replaces body deformation. Indeed it may be that the virtual mass of the driving configuration dominates, so that the recoil could be very one-sided. Vortex shedding may have a significant effect, but an estimate of virtual mass associated with the deformed surface would indicate the contribution to body momentum.

\section{References}

BüHLER, O. 2007 Impulsive fluid forcing and water strider locomotion. J. Fluid Mech. 573, 211-236.

Bush, J. W. M. \& Hu, D. L. 2006 Walking on water: biolocomotion at the interface. Annu. Rev. Fluid Dyn. 38, 339-369.

Bush, J. W. M., Hu, D. L. \& Prakash, M. 2008 The integument of water-walking arthropods: form and function. Adv. Insect Physiol. 34, 117-192.

Hu, D. L. \& Bush, J. W. M. 2010 The hydrodynamics of water-walking arthropods. J. Fluid Mech. 644, 5-33.

Keller, J. B. 1998 Surface tension force on a partially submerged body. Phys. Fluids 10, 3009-3010.

Lighthill, M. J. 1975 Mathematical Biofluiddynamics. Regiional Conference Series in Applied Mathematics, Society for Industrial and Applied Mathematics, vol. 17, Philadelphia, PA, SIAM.

Prakash, M. \& Bush, J. W. M. 2010 Interfacial propulsion by directional adhesion. Nature Materials. (submitted).

Saffman, P. G. 1967 The self-propulsion of a deformable body in a perfect fluid. J. Fluid Mech. 28, 385-389.

Suter, R. B., Loeb, S., Wildman, H. \& Long, J. 1997 Locomotion on the water surface: propulsive mechanism of the fisher spider Dolomedes triton. J. Exp. Biol. 200, 2523-2538. 